\title{
Preliminary Risk Assessment Posed by Formaldehyde Residues in Clothing to Vietnamese Consumers
}

\author{
Van Nam Thai, Akihiro Tokai \\ Osaka University, Division of Sustainable Energy and Environmental Engineering, Osaka, Japan. \\ Email: thai@em.see.eng.osaka-u.ac.jp,namtv78@yahoo.com
}

Received January $19^{\text {th }}, 2011$; revised February $26^{\text {th }}, 2011$; accepted April $6^{\text {th }}, 2011$.

\begin{abstract}
The assessment of potential health risks posed by formaldehyde in clothing to consumers is of increasing concern worldwide. Because of this, it is necessary to develop an exposure model that can realistically mimic clothes wearing conditions. This study aims to preliminarily develop a health risk assessment model for formaldehyde in clothing, and then to assess the potential health risk posed by formaldehyde in textiles to adults and infants in Vietnam using the model. Finally, this study aims to examine the plausibility of the adopted permissible values for formaldehyde in clothing in Vietnam. In the model, two exposure factors for dermal exposure and overall exposure routes, i.e. sweat type and perspiration area, were considered. The margins of exposure (MOE) were calculated to estimate the health risks from worst case and average exposures. The assessment shows that acute exposure via inhalation can pose health risks to Vietnamese consumers in both cases. In regards to chronic exposure, dermal exposure is about four (for infants) and seven (for adults) times higher than exposure via inhalation, but no risks were found for average exposure. If a MOE of 100 is defined as 'safe' used, dermal and total chronic exposure to worst case cause potential risks, whereas no health risks were found for exposure to average case. With the model, the adopted Vietnamese permissible values for formaldehyde in clothing were assessed as not posing a health risk to Vietnamese consumers, hence they are accepted.
\end{abstract}

Keywords: Formaldehyde, Health Risk, Exposure Model, Clothing, Vietnamese Consumers

\section{Introduction}

In 2004, the International Agency for Research on Cancer (IARC) classified formaldehyde as a human carcinogen [1] as well as highly toxic, and irritating. Subsequent to this classification, the first serious exposure event occurred in New Zealand (2007) [2] from Chinese imports. Many countries then carried out analytical studies of formaldehyde in clothes and set limits, e.g. the European Union (EU) (2007) [3], Australia (2007), Netherlands (2008), the US (2008) [4], and the US (2010) [5]. Thus, formaldehyde residue in clothing has become a hot issue for imported textiles.

China Daily (2009) reported that $46.5 \%$ of clothing produced in Guangdong province, the most industrialized province in China, which exports clothing to Vietnam, exceeded the permissible levels of formaldehyde in textiles. To control this substance in imported clothing and textiles (mainly from China, 36.6\% of total imports) (Vietnam Statistics Office, 2009), the Vietnam Ministry of Industry and Trade (MOIT) issued a contemporary regulation for formaldehyde limits [6]. However, there has been no demonstrated research in terms of risk assessments of Vietnamese consumer's health when using such contaminated textiles. In addition, although the adopted permissible values, based on those of the EU Flower Label and Oeko-tex 100, are appropriate to export textiles, the question 'Are they suitable for domestic consumers?' should be quantitatively answered in terms of scientific grounds. Therefore, it is necessary to create a model to identify any health risks to Vietnamese consumers and to check the plausibility of the adopted values.

Some recent studies on formaldehyde in clothing have focused on measuring formaldehyde in clothes (both free and extracted partly through hydrolysis), and then comparing these measurements with standard values [2,5,7]. Others have extensively studied the human health risk assessment from dermal exposure, but there is little information concerning the exposure via inhalation. In gen- 
eral, these studies have not considered certain factors that could influence formaldehyde transfer from clothes into human body, e.g. sweat type [3] or specific contact areas such as respiration zones [8]. The EU Federal In-stitute for Risk Assessment also states that a more realistic estimate of exposure should be developed to include these two factors [9]. Therefore, a developed model for human health risk assessment, not only for formaldehyde residues but also for other hazardous substances (such as heavy metals and dyestuffs) clothing needs to be developed.

In this context, this study aims to (1) develop a health risk assessment model for formaldehyde in clothing that integrates dermal and inhalation exposure and incorporates the factors of sweat type and specific contact areas, (2) assess the potential consumer health risk of formaldehyde in imported textiles, which are stipulated to comply with the regulation issued by MOIT [6] and (3) examine the plausibility of the adopted Vietnamese permissible values for formaldehyde in clothing for infants and adults. To do this, a model with the two factors based on typical characteristics of Vietnamese consumers is first proposed. This model is then applied in assessing the health risk of formaldehyde in imported clothing to Vietnamese consumers and by examining the plausibility of the adopted permissible values of formaldehyde in clothing.

\section{Methods}

\subsection{Framework}

Formaldehyde in clothing poses two key health risks: (1) dermal exposure resulting in allergic contact dermatitis; and (2) chronic inhalation exposure, which may cause cancer $[5,10]$. The method proposed in both the European Technical Guidance Document on Risk Assessment [11] and the Human and Environmental Risk Assessment (HERA) Guidance Document [12], which assumes a percent weight fraction of a chemical being transferred from clothing and absorbed into the skin via dermal exposure, has primarily been used for the estimation of total exposure. For exposure via inhalation, the exposure concentration was limited to the maximum amount according to the ideal gas law. Because of a lack of available relevant data, many studies relied on single-point estimates for the exposure term using average case, worst case or maximum legal values.

In this study, exposure is calculated as a total body dose including dermal and inhalation exposure for different users (infants and adults). Such exposures were then modelled using worst case and average case. The average and worst cases are similar in all respects except for the concentration levels of formaldehyde in clothing. The model differs from the previous research in two respects: (1) it includes the factors of sweat type and specific contact area (respiration zones) for dermal exposure and (2) it is designed for assessing the health risks to Vietnamese consumers. Sweat types and specific contact areas can influence the weight faction of formaldehyde that is transferred and absorbed into skin [9]. For estimating health risks, margins of exposure (MOE), the ratio of no observed adverse effect level (NOAEL) to the actual exposure, selected from previous dose-response assessment bioassays and exposure concentrations were calculated for individual routes and for the total of all routes. Figure 1 schematically shows the framework in detail. In addition, we also assessed the health risk to the maximum permissible legal values for formaldehyde for adults and infants using the proposed model.

\subsection{Data Collection}

When the Directive of Formaldehyde Limits in Imported Textiles was promulgated, from November, 2009 to January, 2010 there were 16 instances of formaldehyde exceeding the limit out of 800 batches sampled. They were mainly exports from China [13]. Formaldehyde is analysed after extraction from clothing by water. Table 1 shows the concentration of formaldehyde in water, $\mathrm{C}_{\text {water, }}$, for 16 samples that exceeded the limit. These values were obtained from the Centre for Standards, Quality and Measurements-Branch 3 in Ho Chi Minh (HCM) City commissioned by the Ministry of Industry and Trade

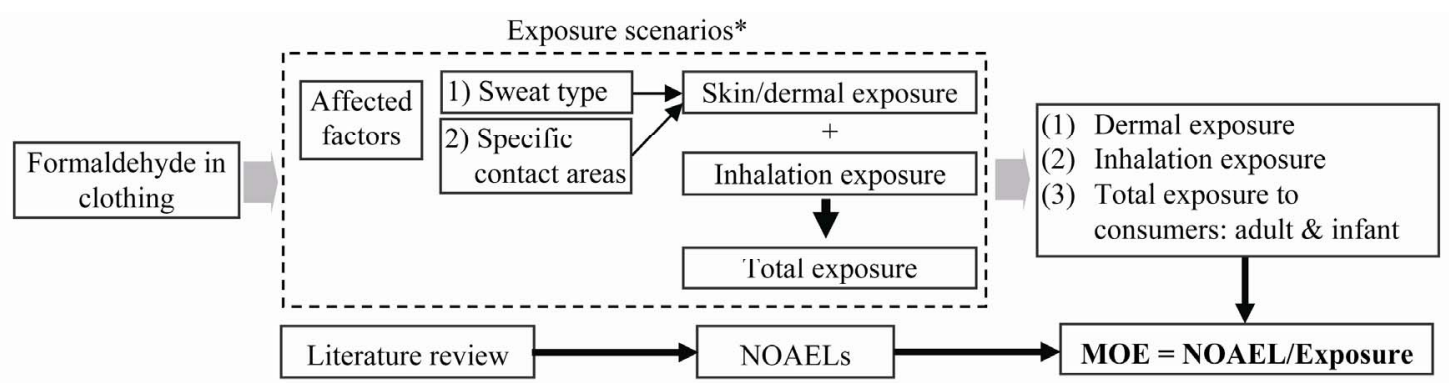

Figure 1. Risk assessment for exposure to formaldehyde in clothing for consumer's health (*Worst case and average point estimates for two exposure routes). 
Table 1. Analytical data for formaldehyde in imported textiles (mg/kg fabrics).

\begin{tabular}{|c|c|c|c|c|c|}
\hline \multirow[t]{2}{*}{ No } & \multicolumn{2}{|c|}{ Nov., Dec., 2009} & \multirow[t]{2}{*}{ No } & \multicolumn{2}{|c|}{ Jan., 2010} \\
\hline & Sample & Value $\left(\mathrm{C}_{\text {water }}\right)$ & & Sample & Value $\left(\mathrm{C}_{\text {water }}\right)$ \\
\hline 1 & F27 & 2,619 & 11 & F623 & 812 \\
\hline 2 & F38 & 750 & 12 & F690 & 1,337 \\
\hline 3 & F65 & 3,517 & 13 & F699 & 463 \\
\hline 4 & F96 & 384 & 14 & F728 & 784 \\
\hline 5 & F99 & 2,035 & 15 & F747 & 359 \\
\hline 6 & F113 & 806 & 16 & F791 & 982 \\
\hline 7 & F357 & 1,334 & & \multicolumn{2}{|c|}{ Formaldehyde limits in textiles [6] } \\
\hline 8 & F389 & 872 & & Infant (<3 year-old) & 30 \\
\hline 9 & F487 & 397 & & Direct contact & 75 \\
\hline 10 & F539 & 537 & & Indirect contact & 300 \\
\hline
\end{tabular}

Mean $=1,124 ;$ Max value $=3,517 ; S D=889$ with the $95 \%$ confidence interval being \pm 474 . Accordingly, formaldehyde concentration $=1,124 \pm 474$ (mg $/ \mathrm{kg}$ textile).

with the support of a recommendation letter from the HCM Environmental Protection Agency.

\subsection{Exposure Scenarios}

To approach the three purposes described in the introduction, a health risk assessment model for dermal and inhalation exposure was first devised. The calculations of the estimated exposures were performed twice: once based on the highest relevant concentrations (i.e. the worst case) and once based on average concentrations (average case) that consumers could be exposed to. Considerations of the individual exposure routes and their respective concentrations, i.e. skin exposure $\left(\operatorname{Exp}_{\text {skin }}\right)$ and inhalation exposure (Expinhalation), led to the expression for the overall exposure (Exp total $)$ shown in Equation (1):

$$
\operatorname{Exp}_{\text {total }}=\operatorname{Exp}_{\text {skin }}+\operatorname{Exp}_{\text {inhalation }}
$$

\subsubsection{Skin Exposure}

Consumers can be directly exposed to formaldehyde dermally by wearing clothing processed with it (permanent press fabrics or anti-wrinkle/crease fabrics). Equation (2), based on the HERA Guidance Document [12], but modified to suit exposure is as follows. (The HERA Guidance Document proposed an exposure model for esterquats, fabric conditioners, that remain in clothes washed and softened with such substances, but not formaldehyde).

$$
\operatorname{Exp}_{\text {skin }}=C \times S \times F D \times F_{1} \times F_{2} \times N / B W
$$

where

Exp $_{\text {skin. }}$ dermal (skin) systemic consumer exposure ( $\mathrm{mg} / \mathrm{kg}$ of body weight/day)
$C$ : formaldehyde concentration in clothing ( $\mathrm{mg} / \mathrm{kg}$ textile) (also called $C_{\text {water }}$ in this paper)

$S$ : surface area of exposed skin $\left(\mathrm{m}^{2}\right)$

$F D$ : fabric density $\left(\mathrm{g} / \mathrm{m}^{2}\right)$

$F_{1}$ : percent weight fraction of formaldehyde transferred from clothing to skin (migration ratio) (\%)

$F_{2}$ : percent weight fraction of formaldehyde absorbed by the skin (penetration ratio) (\%)

$N$ : exposure frequency $\left(\right.$ day $\left.^{-1}\right)$

$B W$ : body weight $(\mathrm{kg})$

\subsubsection{Parameter Estimations}

C (mg/kg) was assigned average and maximum values (worst case) from the measured formaldehyde concentration in Table 1. The total body areas (S) for an adult and a child were assumed to be $18,150 \mathrm{~cm}^{2}$ and $6,700 \mathrm{~cm}^{2}$, respectively [3]. The exposed area was taken to be $85 \%$ of a person's total body area [3], giving $S_{\text {adult }}=15,430 \mathrm{~cm}^{2}$ and $\mathrm{S}_{\text {child }}=5,695 \mathrm{~cm}^{2}$. Cotton and cotton/polyester blended fabrics are the most predominant in Vietnam; hence, the average FD $\left(\mathrm{g} / \mathrm{m}^{2}\right)$ was assigned to be $200 \mathrm{~g} / \mathrm{m}^{2}$ [14]. The bases for the assessment of formaldehyde exposure from clothing are the migration ratio $\left(\mathrm{F}_{1}\right)$ and the penetration ratio $\left(F_{2}\right)$. The value of $F_{2}$ is determined by the octanol water partition coefficient $\left(\mathrm{K}_{\mathrm{ow}}\right)^{1}$. Since $\log \left(\mathrm{K}_{\mathrm{ow}}\right)$ of formaldehyde is 0.35 [1], formaldehyde is categorised as hydrophilic. Based on the HERA Guidance Document $[12]^{2}$, we selected $\mathrm{F}_{1}$ as being $100 \%$ and $\mathrm{F}_{2}$ as being $5 \%$ in normal areas and $10 \%$ in high contact

${ }^{1}$ If a chemical has $\log \left(K_{o w}\right) \in[-3,1)$, it is hydrophilic; $\in[1,4)$, relatively hydrophobic and $\in[4,7]$, very hydrophobic.

${ }^{2}$ Penetration ratios of hydrophilic textile auxiliaries are $5 \%$ and $10 \%$ for normal and respiration zones, respectively. 
areas (perspiration areas) [9]. It was assumed that consumers wear clothes for the entire day $\left(\mathrm{N}=24\right.$ hours) ${ }^{3}$. The standard BW of a 3-year-old child $\left(\mathrm{BW}_{\text {child }}\right)$ was assumed to be $13.9 \mathrm{~kg}$ [15], while the average weight of a Vietnamese adult $\left(\mathrm{BW}_{\text {adult }}\right)$ was calculated as being $56.0 \mathrm{~kg}$. This latter calculation used the following algorithm: $\mathrm{BW}_{\text {adult }}(\mathrm{kg})=\mathrm{BMI} \times \mathrm{H}^{2}$ (BMI: Body Mass Index, average $\mathrm{BMI}=22$; $\mathrm{H}$ : the height of a Vietnamese adult, average $H=1.59 \mathrm{~m} \mathrm{[16]).}$

\subsubsection{Exposure Factors}

Temperature ( $\left.\mathrm{t},{ }^{\circ} \mathrm{C}\right)$ and humidity were assumed to be constants with $t$ being $25^{\circ} \mathrm{C}$. We excluded the $\mathrm{pH}$ of washing water owning to unavailable data and high uncertainty. Ryan et al. [17] stated that areas with high contact with specific parts of the body (specific contact areas or perspiration areas) are the most allergic to formaldehyde. The authors studied diagnosis of allergic contact dermatitis from formaldehyde irritation and observed that the common eruption sites, such as around the neck, the lateral thorax, the flexor surfaces and the waistband were those highly exposed to clothing. It was concluded that these areas can influence the absorption of formaldehyde into skin. To calculate the perspiration areas we utilised into allergic contact dermatitis by Ryan et al. above and the proportions of the skin surface identified by Mathieu [18]. Using these, perspiration areas were estimated to be $30 \%$ of the total exposure area. The penetration ratio in these areas was assigned the variable $\mathrm{F}_{2 \text { sweat }}$.

As described previously formaldehyde is usually extracted by water (refer to $\mathrm{C}_{\text {water }}$ shown in Table 1. However, according to research by the European Commission [3], it is better to mimic the real extraction conditions in which consumer sweat (either acid or basic) extracts the formaldehyde than using water. The research shows that formaldehyde concentrations after extraction by basic/acid sweat solution are on average 1.3 times higher than those after extraction by a water solution. As a result, a better estimate of concentrations of formaldehyde in the $F_{2 \text { sweat }}$ area is $C_{\text {sweat }}=\alpha \times C_{\text {water }}(\alpha=0.8-2.5$ with an average of 1.3, $\alpha=2.5$ in the worst case [3]). Using this, Equation 2 can be written as follows:

$$
\begin{aligned}
\operatorname{Exp}_{\text {skin }}= & \left(C_{\text {water }} \times 0.7 S \times F_{2}+C_{\text {sweat }} \times 0.3 S \times F_{2 \text { sweat }}\right) \\
& \times F D \times F_{1} \times N / B W
\end{aligned}
$$

\subsubsection{Inhalation Exposure}

Exposure via inhalation is expressed as the concentration of formaldehyde in the air in a breathing zone and is given as an average concentration over a reference period. The vapour immediately produces local irritation in $\mathrm{mu}-$

${ }^{3}$ Actually, consumers can change their clothes, but it is assumed that $S$ and FD are constants. cous membranes, including the eyes, nose and upper respiratory tract (acute exposure) $[1,17]$ and recently it has been reported that inhaled formaldehyde may cause cancer (from chronic exposure) [5]. Because buildings in Vietnam commonly have minimal use of external windows and openings, and poor natural and mechanical ventilation [19], the ventilation of a given room is assumed to be insufficient. It is also assumed that formaldehyde is released instantaneously to the entire room and distributed homogenously. Equations (4)-(6) are based on the European Technical Guidance Document on Risk Assessment [11], modified to suit clothing contact and were used to estimate formaldehyde entering the body via inhalation. For the calculation of theoretical maximum concentration of formaldehyde in the air $\left(C_{\text {vapour }}\right)$, the ideal gas law was utilised (see Equation 6).

$$
\begin{gathered}
\operatorname{Exp}_{\text {inhalation }}=C_{\text {inh }} \times V_{\text {air breathed }} \times F_{3} \times n / B W \\
C_{\text {inh }}=C_{\text {vapour }} \times \frac{Q_{\text {clothing }}}{V_{\text {room }}} \\
C_{\text {vapour }}=C_{\text {water }} \times \frac{M W}{22.4} \times \frac{273}{T E M_{a}} \times \frac{P_{a}}{101325}
\end{gathered}
$$

where

Exp $_{\text {inhalation: }}$ formaldehyde intake to the body via mucous membranes (mg/kg of body weight/day)

$C_{\text {inh }}$ : formaldehyde concentration in air at specific sites $\left(\mathrm{mg} / \mathrm{m}^{3}\right)$

$V_{\text {air breathed }}$ : the volume of air that a person breathes per day; $15 \mathrm{~m}^{3}$ for an adult, $6 \mathrm{~m}^{3}$ for a child [20]

$F_{3}$ : fraction of formaldehyde inhaled or respired (\%) $\left(\mathrm{F}_{3}=100 \%\right)[10,21]$

$n$ : exposure frequency; according to the study by Shin et al. [22] an adult stays indoor for 20 hours (13.7 hours at home, 6.4 hours for working) while a child is also in a room for 20 hours (8 hours at home, 12 hours at school). Assuming that if they are outdoor, there is no effect by inhalation of formaldehyde from clothing. Hence, $\mathrm{n}_{\text {adult }}=$ 20/24 $\left(\right.$ day $\left.^{-1}\right)$ and $n_{\text {child }}=20 / 24\left(\right.$ day $\left.^{-1}\right)$.

$B W$ : body weight $(\mathrm{kg}) . B W_{\text {child }}=13.9 \mathrm{~kg}$ and $B W_{\text {adult }}=$ $56 \mathrm{~kg}$ (see Section 2.3.1).

$Q_{\text {clothing }}=\mathrm{S} \times \mathrm{FD}(\mathrm{kg})$; hence, $Q_{\text {clothing }}$ for adults and children is $0.31(\mathrm{~kg})$ and $0.11(\mathrm{~kg})$, respectively.

$C_{\text {vapour }}$ : concentration of formaldehyde vapours from clothing at the examined temperature of $25^{\circ} \mathrm{C}(\mathrm{mg} / \mathrm{kg})$. It is estimated from $C_{\text {water }}$ (the concentration of formaldehyde in clothing as shown in Table 1).

$V_{\text {room: }}$ : the volume of a closed room in which people stay/work; $30 \mathrm{~m}^{3}$ for an adult, $18 \mathrm{~m}^{3}$ for a child $\left(\mathrm{m}^{3}\right)$ [8].

$M W$ : molecular weight of formaldehyde (30.03 g/mole); $\mathrm{TEM}_{\mathrm{a}}$ and $\mathrm{P}_{\mathrm{a}}$ are the actual temperature in $\mathrm{K}$ and the vapour pressure in Pascals $\left(3,466.4 \mathrm{~Pa}\right.$ at $\left.25^{\circ} \mathrm{C}\right)$ for formaldehyde, respectively [1]. 


\subsection{Acute and Chronic Exposures}

Many people have a habit of wearing new clothing without washing it first $[23,24]$. Accordingly, we distinguished between acute exposure while wearing new clothes with a possibly higher migration rate and chronic exposure during the whole course of usage. Based on Equations (3) and (4), we first estimated acute exposure by using the first migration data, and then undertook a risk assessment of acute exposure and allergic reactions. Chronic exposure depends on experimental tests using simulated wash, wear cycles, $\mathrm{pH}$ of detergents, etc, which are not readily available. Hence, alternatively, to determine chronic exposure for the risk assessment of chronic toxicity, the Danish Ministry of Environment [8] and the EU Federal Institute for Risk Assessment [9] recommend using a safety factor of one-tenth (1/10) of the acute exposure estimates. This study concentrates on both acute and chronic exposures.

\subsection{Preliminary Risk Assessment}

As mentioned in the method section, MOEs can be used to assess whether formaldehyde contained in clothing has a potential adverse health effect when using such clothing. To do this, data on NOAEL has been selected from available literature, where the focus has typically been on the skin's ability to absorb formaldehyde and inhalation of it through mucous membranes (via the eyes, throat and nose). In this study, the two lowest NOAELs were used, namely $\mathrm{NOAEL}_{\text {oral (chronic) }}=10$ (mg/kg.day) established on the basis of a 24-month oral toxicity study of total exposure (including dermal and inhalation routes) and NOAEL $_{\text {inhalation (acute) }}=1.3\left(\mathrm{mg} / \mathrm{m}^{3}\right)$ based on a 3-consecutive-day inhalation exposure [25]. The latter was also considered because many studies show that the inhalation of formaldehyde can immediately cause local irritation in mucous membranes when consumers are in contact with formaldehyde. By using MOEs it is possible to assess the human health risk to consumers from formaldehyde in monitored clothing. The values of MOEs are calculated by Equation7, i.e. $\mathrm{MOE}_{\text {total }}=\mathrm{NOAEL}_{o}$ ral $/$ Exp $_{\text {total }}$ while $\mathrm{MOE}_{\text {inh }}=\mathrm{NOAEL}$ inhalation $/ \mathrm{Exp}_{\text {inhalation }}$ [26].

$$
M O E=N O A E L / E x p
$$

\section{Results and Discussion}

\subsection{Assessment of Human Health Risk from Formaldehyde}

From the monitored concentrations of formaldehyde $\left(\mathrm{C}_{\text {water }}\right)$, we estimated $\mathrm{C}_{\text {sweat }}$ for dermal exposure and $\mathrm{C}_{\mathrm{va}}$ pour for exposure via inhalation.

The results of the exposure calculations are given in Table 2 for dermal, inhalation and total exposure. Dermal exposure is about four times (for infants) to seven times (for adults) higher than exposure via inhalation, meaning dermal exposure is the dominant route. This result agrees with the existing medical literature, i.e. the greatest concern for human health associated with formaldehyde in clothing is allergic contact dermatitis that stems from dermal exposure [5].

It is apparent that the potential dermal exposure of a child is higher than that of an adult owning to lower BW of children. The results show that the average (and worst case) dermal uptakes were 0.46 (2.13 worst case) and 0.68 (3.17 worst case) $\mathrm{mg} / \mathrm{kg}$ bw/day for an adult and for a child, respectively. These exposures are similar to those in the worst case research by Ellebak et al. for Danish consumers, i.e. 0.31 (adult) and 1.10 (child) $\mathrm{mg} / \mathrm{kg}$ bw/day [27] and slightly lower than those in a study for European consumers $(1.2$ and $3.1 \mathrm{mg} / \mathrm{kg}$ bw/day, respect- tively, in the worst case). However, the maximum dose of formaldehyde in imported clothing in Vietnam $(3,517 \mathrm{mg} / \mathrm{kg})$ is very much higher than that in Europe $(162.5 \mathrm{mg} / \mathrm{kg})$. This difference is because the research for Dan- ish and European consumers used $\mathrm{F}_{2}=100 \%$ owning to absence of data, whereas we used $\mathrm{F}_{2}=10 \%$ for perspire- tion zones and $5 \%$ for other zones based on the latest discussion for garment textiles of the EU Federal Institute for Risk Assessment [9] and the fact that that the weights of Vietnamese people are less than those of Europeans. Regarding exposure via inhalation, estimated formaldehyde concentrations in the room sizes assumed are higher than the recommended threshold limit value (TLV) for indoor conditions $\left(0.15 \mathrm{mg} / \mathrm{m}^{3}\right)$ [8] by three times in the average case and ten times in the worst case. Rumchev et al. [28] state that children exposed to a formaldehyde level of $\geq 60 \mu \mathrm{g} \cdot \mathrm{m}^{-3}$ are at increased risk of contracting asthma; in contrast, the average inhalation exposure for children in

Table 2. Worst case and average exposure estimates for users via each route and as a total.

\begin{tabular}{|c|c|c|c|c|c|c|c|c|}
\hline \multirow{2}{*}{$\begin{array}{l}\text { Route } \\
\text { User }\end{array}$} & \multicolumn{2}{|c|}{ Inhalation (mg/m³) } & \multicolumn{2}{|c|}{ Inhalation (mg/kg/day) ${ }^{1}$} & \multicolumn{2}{|c|}{ Dermal (mg/kg/day) ${ }^{2}$} & \multicolumn{2}{|c|}{ Total (mg/kg/day) ${ }^{1+2}$} \\
\hline & Adult & Child & Adult & Child & Adult & Child & Adult & Child \\
\hline Average & $0.49 \pm 0.21$ & $0.29 \pm 0.12$ & $0.11 \pm 0.05$ & $0.10 \pm 0.04$ & $0.46 \pm 0.19$ & $0.68 \pm 0.29$ & $0.57 \pm 0.24$ & $0.78 \pm 0.33$ \\
\hline Worst case & 1.53 & 0.90 & 0.34 & 0.32 & 2.13 & 3.17 & 2.47 & 3.49 \\
\hline
\end{tabular}

Values in this table are acute exposures. Chronic exposures are estimated to be one-tenth of acute exposures 
this study for children was about $290 \mu \mathrm{g} \cdot \mathrm{m}^{-3}$, five times higher than the level suggested by Rumchev.

With respect to chronic exposure, almost all exposure routes and users have MOEs much larger than 1, even for the worst cases. Most exposure is again via the dermal route. For acute exposure, e.g. consumers wearing new clothes without washing, after ironing or hot washing (which can generate free formaldehyde from formaldehyde carriers), MOEs are around 1, suggesting potential health risks, e.g. histopathological effects or increased cell proliferation in the nasal cavity. Since formaldehyde is highly absorbed in the respiratory and gastro-intestinal tracts [29], acute exposure via inhalation plays an important role in assessing the health risk of formaldehyde. To reduce the risk of acute inhalation exposure, washing new clothes (which results in a $90 \%$ decrease in formaldehyde levels after one wash and a further decrease to $5 \%$ of original levels after several washes [30] and/or living in a well-ventilated room [22,28,31] are two of the best solutions that past studies have demonstrated. For chronic exposure, some studies report that while formaldehyde levels may decline initially after washing, the levels may start increasing again after multiple washes. This can be explained by noting that during washing and ironing, resins fixed on clothes are broken down, becoming more ingrained in the fabrics [5]. This underpins the assumption that one is exposed to formaldehyde dermally and via vapours in the room during the longterm exposure.

Average and worst case exposure approaches such as those in this research are often used for the screening of risk. Estimated MOEs are only a rough guide for assessing the health risks of formaldehyde. It has to be taken into account that exposure levels obtained from such approaches might be 10 to 100 lower than the actual exposure, i.e. the MOEs estimated in this research might be 10 - 100 higher than they actually are [32,33]. A MOE of 100 is considered for defining a safe level in risk assessment [34] because of inter- and intra-specie variations or any inherent uncertainty in databases. If a MOE of 100 is considered as 'safe', then one can examine Table 3 and see that for chronic exposure via inhalation, even in the worst case, and chronic dermal exposure in average cases poses no potential risks. However, the further research on the accumulation of formaldehyde in the human body should be considered to understand when formaldehyde could cause potential health risk. On the other hand, especially for the worst cases of dermal and total exposure, they are potential health risks. In short, the clothing examined in this study could cause local irritation in mucous membranes, including the eyes, nose and upper respiratory tract (owning to acute exposure via inhalation), and create health risks for Vietnamese con- sumers with chronic dermal exposure to the worst case concentrations.

The advantage of the proposed model is that two factors, perspiration zones and the sweat type, which were not modelled in the previous studies, are included. Furthermore, the model deals with the penetration ratio based on latest studies. In addition, the model combines two exposure routes (inhalation and dermal exposure), whereas past research in the EU, New Zealand and Denmark only considered dermal exposure. However, the research presented on this paper has some limitations: (1) it only uses point estimates and (2) it assesses health risks associated with imported clothing only. The former should be replaced by probabilistic estimates where sufficient information is available and the latter by domestically made clothes. With such enhancements, a more detailed and comprehensive picture of the health risk associated with formaldehyde in clothing could be made. However, since this study is the first study in terms of health risk of household products in Vietnam, the lack of relevant data has limited this research.

The validity of the proposed model comes from the fact that it is based on exposure models for health risk assessment developed by the European Commission (EC, 2003). The latest research by the EC on the release of formaldehyde from textiles [3] concludes that it would be better to mimic real textile usage conditions by replacing the present water extraction analysis of formaldehyde with a modified method using artificial perspiration solutions. This was the reason that we changed $\mathrm{C}_{\text {water }}$ to $\mathrm{C}_{\text {sweat }}$ for the perspiration zones, thereby describing more realistic conditions. We also based the model on the latest dermatological studies $[17,18]$ to identify the perspiration zones where there is a high risk of irritation caused by formaldehyde. The usage of a more acute penetration ratio $\left(\mathrm{F}_{2}\right)$ and inhalable ratio $\left(\mathrm{F}_{3}\right)$-as compared to past research is also appropriate, as exposure studies state that close to $100 \%$ of formaldehyde is readily absorbed in the respiratory and gastro-intestinal tracts while dermal absorption of formaldehyde appears to be less [29].

\subsection{Plausibility of Adopted Legal Values of Formaldehyde}

The third purpose of this study is to examine the plausibility of the adopted Vietnamese permissible values for formaldehyde exposure for adults and children. They are set in terms of maximum legal values, being $\mathrm{C}_{\text {child }}=30$ and $\mathrm{C}_{\text {adult }}=75$ (mg/kg textile) [6] (for comparison to actual values, see Table 1). Exposure estimates and MOE values derived from the maximum legal permissible concentration are shown in Table 4.

The results in Table 4 show that there should be no health problems connected with chronic exposure to the 
Table 3. MOEs based on worst case and average exposure estimates by each route and as a total of all routes.

\begin{tabular}{lrrrrrrrr}
\hline Route & \multicolumn{2}{c}{ Inhalation (acute) } & \multicolumn{2}{c}{ Inhalation (chronic) } & \multicolumn{2}{c}{ Dermal (chronic) } & \multicolumn{2}{c}{ Total (chronic) } \\
User & Adult & Child & Adult & Child & Adult & Child & Adult & Child \\
\hline Average & $\mathbf{2 . 6}$ & $\mathbf{4 . 5}$ & 909 & 1,000 & 217 & 147 & 175 & 128 \\
Worst case & $\mathbf{0 . 8 5}$ & $\mathbf{1 . 4}$ & 294 & 312 & 47 & 32 & 40 & 29 \\
\hline
\end{tabular}

MOE $=$ NOAEL/Intake (exposure)

Table 4. Exposure estimates (chronic) and MOE values based on maximum legal values.

\begin{tabular}{cccccccccc}
\hline \multirow{2}{*}{ User } & \multicolumn{4}{c}{ Exposure $\left(\mathrm{mg} / \mathrm{kg}\right.$.day) or concentration $\left(\mathrm{mg} / \mathrm{m}^{3}\right)$} & & \multicolumn{2}{c}{ MOE } \\
\cline { 2 - 9 } & Inhalation $_{\text {acute }}$ & Inhalation & Dermal & Total & Inhalation acute & Inhalation & Dermal & Total \\
\hline Adult & 0.03 & 0.007 & 0.046 & 0.053 & 43 & 14,286 & 2,174 & 1,887 \\
Child & 0.01 & 0.003 & 0.027 & 0.030 & 130 & 33,333 & 3,704 & 3,333 \\
\hline
\end{tabular}

MOE = NOAEL/Intake (exposure); Inhalation $_{\text {acute }}\left(\mathrm{mg} / \mathrm{m}^{3}\right)$

maximum permissible legal concentrations, as the MOEs are far greater than 100 . Furthermore, the corresponding legal maximum formaldehyde concentrations for adults and children are 0.03 and $0.01 \mathrm{mg} / \mathrm{m}^{3}$, respectively for acute inhalation, which are 5 and 15 times below the TLV of $0.15 \mathrm{mg} / \mathrm{m}^{3}$, respectively. In other words, the adopted permissible values of formaldehyde in clothing for Vietnamese consumers are justified.

It should be remembered, however, that formaldehyde can be found in numerous consumer products besides clothing, for example, other textiles such as carpet and curtain, disinfectants, pressed wood, paper, etc. Formaldehyde vapours can be given off by any of these products, and therefore it is necessary to assess and establish total permissible formaldehyde exposure levels. In this context, the permissible level of formaldehyde vapours from clothing would be less than the present values. For example, Japan has the most stringent limits on formaldehyde in infant clothing, i.e. $20 \mathrm{mg} / \mathrm{kg}$ textile [4]. The proposed model could be adapted to include the contributions of all major sources of formaldehyde and establish new limits in Vietnam.

\section{Conclusions and Implications}

This study serves as a preliminary risk assessment to Vietnamese consumers from formaldehyde in clothing. The proposed model could be applied to assess the health risk from other chemicals in clothing as well, such as dyestuffs and heavy metals if the relevant data is available.

The assessment of risks caused by formaldehyde in imported clothing carried out by using the model shows that the potential risk of overall chronic exposure stems mainly from the dermal route. For average exposure, the chronic total exposure (inhalation and dermal exposure) does not pose a risk to Vietnamese consumers, whereas acute exposure could pose a risk if a MOE of 10 or higher are needed. For worst case exposure (with a MOE of 100) dermal and total exposure could cause potential health problems for Vietnamese consumers. In addition, the acute exposure via inhalation can also pose potential health risks. By utilizing the model, the adopted permissible values of formaldehyde in clothing for children and adults are assessed not to pose any health risks and are considered acceptable for Vietnamese consumers.

\section{Acknowledgements}

We express our gratitude to the members of the Department of Environmental Management in HoChiMinh City, who helped with the data collection. Special thanks are due to the Rotary Foundation for the Ph.D. course in Osaka University, Japan.

\section{REFERENCES}

[1] Formaldehyde Council, "Formaldehyde: Facts and Background Information,” 2007. http://www.formaldehyde.org/_base/pdf/fact_sheets/11_0 1_07-FormadehydeFactsandBackgroundInformation.pdf

[2] New Zealand Ministry of Consumer Affairs, "Evaluation of Alleged Unacceptable Formaldehyde Levels in Clothing,” New Zealand, 2007.

[3] European Commission (EC), "European Survey on the Release of Formaldehyde from Textiles,” Europe, 2007.

[4] C. D. G. Anton et al, "Formaldehyde-releasers: Relationship to Formaldehyde Contact Allergy. Formaldehydereleasers in Cloths: Durable Press Chemical Finishes_Part 1,” Contact Dermatitis, Vol. 62, No. 5, 2010, pp. 257-271.

[5] United States Government Accountability Office (GAO), "Formaldehyde in Textiles," Report to Congressional 
Committees, 2010

[6] Vietnamese Ministry of Industry and Trading, "The Contemporary Regulation of Limits of Formaldehyde and Aromatic Amines Releasing from Dyes in Import Textiles,” Vietnam, 2009.

[7] A. J. Scheman, P. A. Carroll, K. H. Brown and A. H. Osburn, "Formaldehyde-Related Textile Allergy: An Update," Contact Dermatitis, Vol. 38, No. 6, 1998, pp. 332-336. doi:10.1111/j.1600-0536.1998.tb05769.x

[8] L. H. Paul et al., "Survey and Health Assessment of Chemical Substances in Hobby Products for Children," Survey of Chemical Substances in Consumer Product, No. 93, 2008.

[9] EU Federal Institute for Risk Assessment, "Introduction to the Problems Surrounding Garment Textiles,” 2007.

[10] The Danish EPA, "Surveys on Chemicals in Consumer Products. Screening for Health Effects from Chemical Substances in Textile Colorants,” Survey, No. 57, 2005.

[11] European Commission (EC), "Technical Guidance Document on Risk Assessment in Support of Directive 93/67/EEC on Risk Assessment for New Notified," Europe, 2003.

[12] Human and Environmental Risk Assessment (HERA), "Esterquats-Human Health Risk Assessment Report,” 2009.

[13] VietChina Business, 2010.

http://www.vietchinabusiness.vn/i-sng/tieu-im/15213-vainhiem-doc-vao-viet-nam

[14] V. N. Thai, A. Tokai and D. T. Nguyen, "Eco-Labeling Criteria for Textile Products with the Support of Textile Flows: A Case Study Of The Vietnamese Textile Industry,” Journal of Sustainable Energy \& Environment, In reviewing.

[15] WHO, "The Table of Height and Weight of Under-5Year-Old Children,” 2008.

http://www.choicungbe.com/tre-thong-minh/bang-chieu-c ao-va-can-nang-chuan-o-tre-duoi- 5-tuoi.ccb

[16] Vietnam National Institute of Physical Science, "Survey Of The Height Of Vietnamese People,” Vietnam, 2010.

[17] M.C. Ryan Et Al., "Diagnosis and Treatment of Dermatitis Due to Formaldehyde Resins in Clothing," American Contact Dermatitis Society, Vol. 15, No. 4, 2004, pp. 169-175.

[18] B. Mathieu Et Al., "Proportion of Skin Surface of Children and Young Infants from 2 to 18 Years Old,” Journal of Investigative Dermatology, Vol.128, 2008, pp. 461-464.

[19] T.H. Dinh, “Current Status of Existing Condominium Buildings in Vietnam: Common Problems, Main Causes and Proposed Maintenance Strategies," International Conference on Sustainable Architectural Design and Urban Planning, Hanoi, 2007.

[20] R. Raghunath Et Al., "Retention Times of Pb, Cd, Cu and Zn in Children'S Blood," the Science of the Total Environment, Vol. 207, No. 2-3, 1997, pp. 133-139. doi:10.1016/S0048-9697(97)00255-6

[21] United States Department of Energy, “Guidance for Conducting Risk Assessment and Related Risk Activities for DOE-ORO Environmental Management,” DOE/BJC/OR
$-271,1999$.

http://risk.lsd.ornl.gov/homepage/bjc or-271.pdf

[22] Y. Shin, D. Lee, D. Won and H. Lim, "Risk Management of Hazardous Chemicals Considering Interaction between Indoor and Outdoor Rources,” Korea Environment Institute, 2007.

[23] Everyday tips and thoughts, "Make Sure You Wash Those Clothes after You buy Them and before You Wear Them," 2010

http://everydaytipsandthoughts.com/health-and-fitness/ma ke-sure-you-wash-those-clothes-after-you-buy-them-andbefore-you -wear-them/

[24] H. Jill, "Wash Clothes before You Wear Them,” Fashion, Science \& Technology, 2010.

http://www.neatorama.com/2010/01/21/wash-clothes-befo re-you-wear-them/

[25] World Health Organization, “Formaldehyde,” Concise International Chemical Assessment Document 40, 2002.

[26] H. Stefan et al., "Consumer Exposure to Biocides-identification of Relevant Sources and Evaluation of Possible Health Effects,” Environmental Health, Vol. 9, 2010, p. 7. doi:10.1186/1476-069X-9-7

[27] L. S. Ellebak et al., "Survey of Chemicals in Consumer Products,” Danish Ministry of Environment and Energy, No. 23, 2003.

[28] K. B. Rumchev, J. T. Speckett, M. K. Bulsara, M. R. Phillips and S. M. Stick, Domestic Exposure to Formaldehyde Significantly Increases the Risk of Asthma in Young Children, European Respiratory Journal, Vol. 20, No. 2, 2002, pp. 403-408. doi:10.1183/09031936.02.00245002

[29] Chemical Safety Information from Intergovernmental Organizations, "Environment Health Criteria 89-Formaldehyde,” 2010. http://www.inchem.org/documents/ehc/ehc/ehc89.htm

[30] National Institute for Public Health and Environment, "Allergens in Consumer Products," RIVM Report 320025001/2008, 2008.

[31] M. H. Sherman and A. T. Hodgson, "Formaldehyde as a Basis for Residential Ventilation Rates,” Lawrence Berkeley National Laboratory, Canada, 2002.

[32] S. Hahn et al., "Health Risks from Biocide-containing Products and Articles of Daily Use," Final Report Research Project 20461 218/05 on Behalf of the German Federal Environmental Agency, 2005.

[33] M. P. Van Veen, J. G. M. van Engelen and M. T. M. van Raaij, "Crossing the River Stone by Stone: Approaches For Residential Risk Assessment For Consumers,” Annual Occupational Hygiene, Vol. 45, No. S1, 2000, pp. 107-118. doi:10.1016/S0003-4878(00)00101-0

[34] WHO, “Assessing Human Health Risks of Chemicals: Derivation Of Guidance Values For Health-based Exposure Limits,” EHC 170 World Health Organization, Geneva, 1994. http://www.inchem.org/documents/ehc/ehc/ehc170.htm. 